\title{
Polarization Effects of Electro-optic Sampling and Over-rotation for High Field THz Detection
}

\section{Gregory Bell ${ }^{1}$ id . Michael Hilke ${ }^{1}$}

Received: 6 February 2020 / Accepted: 18 June 2020 / Published online: 4 July 2020

(C) Springer Science+Business Media, LLC, part of Springer Nature 2020

\begin{abstract}
With ever increasing availability of terahertz fields, it is important to find suitable detection techniques without compromising the measured dynamic range. Electrooptic terahertz sampling techniques, which are commonly used to detect terahertz fields, exhibit over-rotation at high fields that limit the detection accuracy. Here we discuss a method to correct for over-rotation that put no limits on measured terahertz field strengths, while preserving the low field sensitivity. We further evaluate the induced polarizations at high terahertz fields and show how over-rotation can be corrected by simultaneously measuring the polarizations before and after the quarter wave plate.
\end{abstract}

Keywords Terahertz $\cdot$ Electro-optic sampling $\cdot$ Over-rotation $\cdot$ Polarization

\section{Introduction}

Terahertz (THz) spectroscopy [1]-including time-resolved $\mathrm{THz}$ spectroscopy (TRTS), terahertz time-domain spectroscopy (THz-TDS), and $\mathrm{THz}$ emission spectroscopy-is concerned with what frequencies of a $\mathrm{THz}$ field emanate from a sample of interest. Planken and co-workers have pioneered the use of electro-optic (EO) crystals for the detection of THz signals [2]. However, with the advent of ever more powerful $\mathrm{THz}$ fields, effects such as over-rotation [3] can limit the applicability of EO crystals. Moreover, the approximations used in Planken have to be modified for higher fields. This is particularly timely with the ever increasing THz fields. Over two decades ago, $\mathrm{THz}$ field levels at the focal point were already reaching values in the hundreds of $\mathrm{kV} / \mathrm{cm}[4,5]$. A decade later, these field levels were reached without focusing [6-8]. Indeed, the use of high $\mathrm{THz}$ fields, $\geq 1 \mathrm{MV} / \mathrm{cm}$ [9], has seen

Gregory Bell

gregory.bell@mcgill.ca

1 Department of Physics, McGill University, Montreal, Canada 
increased usage in recent years, with beautiful experiments on inducing insulator-tometal transitions in a metamaterial [10] and electronic and magnetic excitations in a ferromagnet [11]. Other experiments include high THz field induced superconductivity $[12,13]$, THz field-induced ferroelectric phases [14, 15], photocarrier dynamics in monolayer graphene [16, 17], and damage to thin metal films [18]. The number of high field $\mathrm{THz}$ emitters is also increasing over time, such as from a thin foil interaction [19], from metal wires [20], and from organic crystals [21]. Sources are reaching field strengths up to the 10's of MV/cm [22-24]. Hence, properly characterizing high field $\mathrm{THz}$ polarization becomes paramount.

Laser probe beam polarization is at the heart of $\mathrm{THz}$ signal detection. The intensity difference in the horizontal and vertical components of the beam relay the electric field strength and direction of the THz signal. For instance, in a naive depiction of the polarization of the probe beam due to EO sampling, where an elliptical polarization is created after the quarter-wave plate (QWP) with the major or minor axis at $45^{\circ}$ from the vertical $[25,26]$, this would not show any sign of THz. This is based on the vertical and horizontal components of the probe beam being not equal, and an ellipse at $45^{\circ}$ has equal vertical and horizontal components. This illustrates the importance to evaluate the complete polarization behaviour and this for the full range of EO sampling, which we present below.

The quasi-static THz electric field creates a changing waveplate in the EO crystal for the laser probe beam pulses. The phenomenon of creating a controllable waveplate in an EO crystal with an electric field due to non-linear optics has been known for decades [27]. The indexes of refraction are altered in different directions, causing the polarization of the beam to change. If a linearly polarized $\mathrm{THz}$ wave enters an EO crystal at $\theta=90^{\circ}$ (angle $\theta$ is shown in Fig. 1), and the indexes of refraction are altered so their difference in the horizontal $\theta=90^{\circ}$ and vertical $\theta=0^{\circ}$ directions is increased, this would have no effect on the polarization-only the speed of the beam may change. To get the maximum change in polarization, the fast axis of the waveplate would have to be at $\theta=45^{\circ}$. This angle corresponds to the angle that the fast axis of the EO crystal is at (after taking axis rotations into account as described in ref. [2]), which the $\mathrm{THz}$ field creates when it also is horizontally linearly polarized. This may seem counter-intuitive with both probe beam and $\mathrm{THz}$ beam linearly polarized in the same direction. However, lack of symmetries in the crystals makes this fast axis at $\theta=45^{\circ}$ created by the $\mathrm{THz}$ possible.

In this work we present a detailed analysis of the changes in polarization in the probe beam in the presence of the $\mathrm{THz}$ field and the $\mathrm{EO}$ crystal at all $\mathrm{THz}$ field intensities. We discuss how the information of the polarization before and after the quarter wave plate (QWP) can be used to correct for issues such as over-rotation without decreasing the dynamic range.

Figure 1 shows a typical EO sampling setup, including an EO crystal (commonly $\mathrm{ZnTe}$ ) where the probe and $\mathrm{THz}$ beams meet, a QWP, a Wollaston prism (a polarizing beamsplitter-PBS), and two balanced photodiodes (PD A and PD B) [25]. Here we assume the probe beam and $\mathrm{THz}$ pulse are p-polarized (horizontal in the lab frame if the beam stays at the same height level). The reason the QWP is needed is so the THz field can be mapped out with the probe beam for "positive" and "negative" directions (the distinctions are arbitrary and can be considered to correspond to "left" 


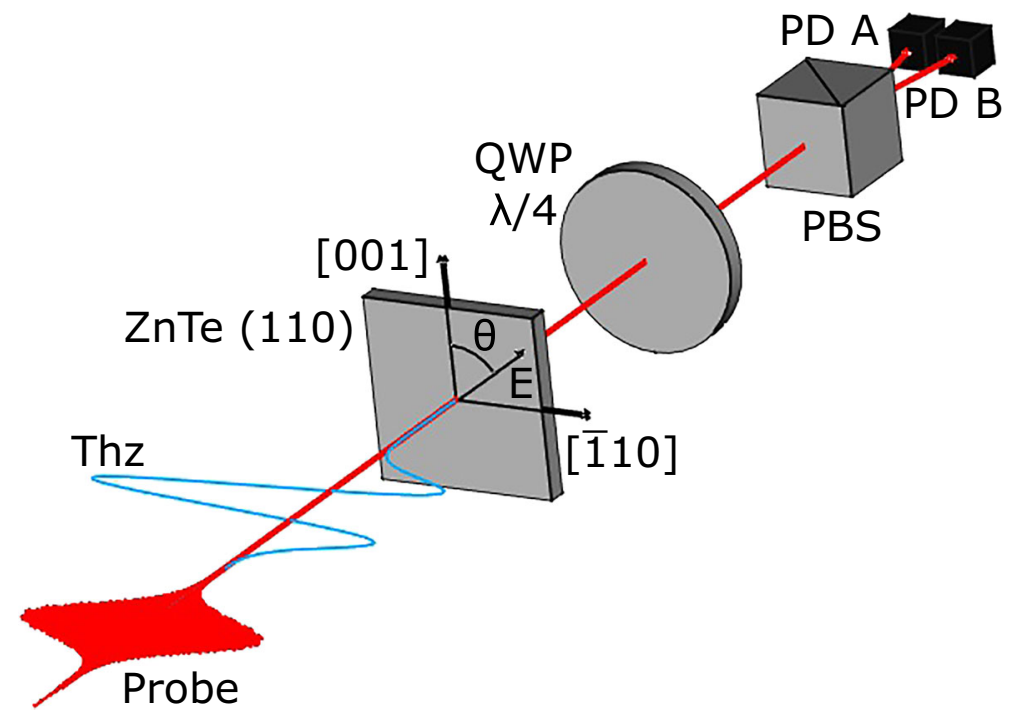

Fig. 1 EO sampling setup, with electric field (E) angles $\theta$. The THz meets with the probe beam in the ZnTe crystal, with the QWP used to adjust polarization of the probe, the PBS to split the vertical and horizontal components of the beam, and the PD's to detect the intensities of each polarization

and "right" in the horizontal plane). With no THz, the probe continues to be linearly polarized after the EO crystal, and becomes circularly polarized after the QWP, as seen in Fig. 2a.

When $\mathrm{THz}$ is present, the probe beam becomes elliptical before and after the QWP, as seen in Fig. 2b. However, when the $\mathrm{THz}$ wave (now considered negative) causes an equal magnitude phase change in the opposite direction, as seen in Fig. 2c, the polarization before the EO crystal looks identical to the previous phase change. After the QWP, the horizontal and vertical intensities $\left(I \propto E^{2}\right)$ are different with these different phase changes. Here we can consider $E_{0^{\circ}}>E_{90^{\circ}}$ as positive, and $E_{90^{\circ}}>$ $E_{0^{\circ}}$ as negative, representing opposite directions of $\mathrm{THz}$ field.

\section{Methods}

Polarization simulations can further display results for greater $\mathrm{THz}$ electric field induced phase changes. When the phase change is $\pi / 2$ or greater, over-rotation occurs. As seen in Fig. $3 \mathrm{~b}$, the $E_{0^{\circ}}$ value after the QWP has reached a maximum. However, the polarization before the QWP is circularly polarized. This means that its $E_{0^{\circ}}$ value can continue to increase. Figure 3 a and c show the polarizations before and after over-rotation, respectively. The polarizations after the QWP are identical, but the polarizations before the QWP are not. This information can be used to correct for over-rotation.

The polarization before the QWP will also reverse at high enough $\mathrm{THz}$ fields, when the phase change is at $\pi$. As seen in Fig. $4 \mathrm{~b}$, the $E_{0^{\circ}}$ amplitude before the QWP 
(a)

Polarization after EO crystal
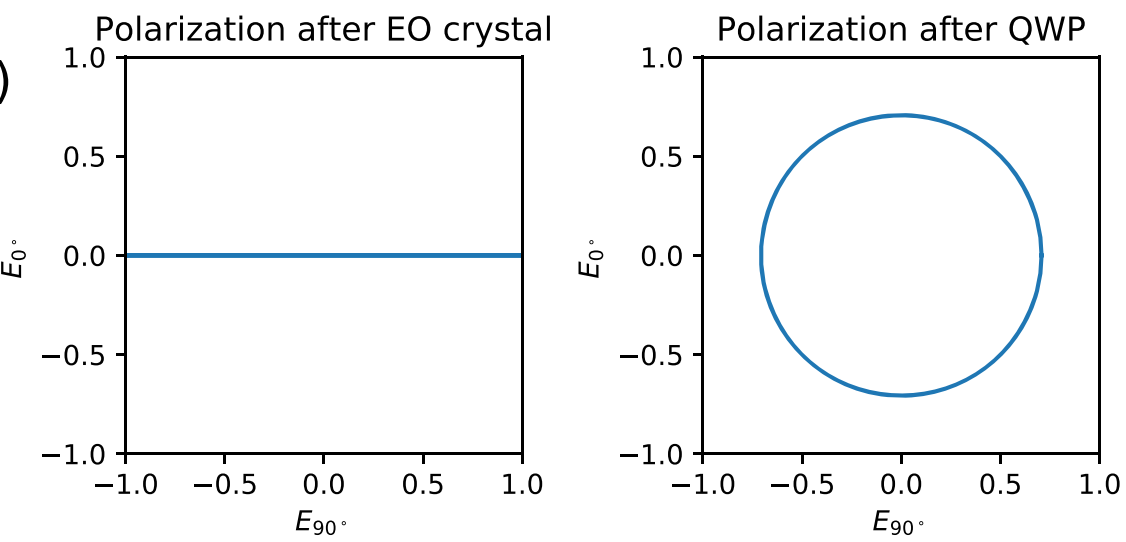

Polarization after EO crystal

(b)
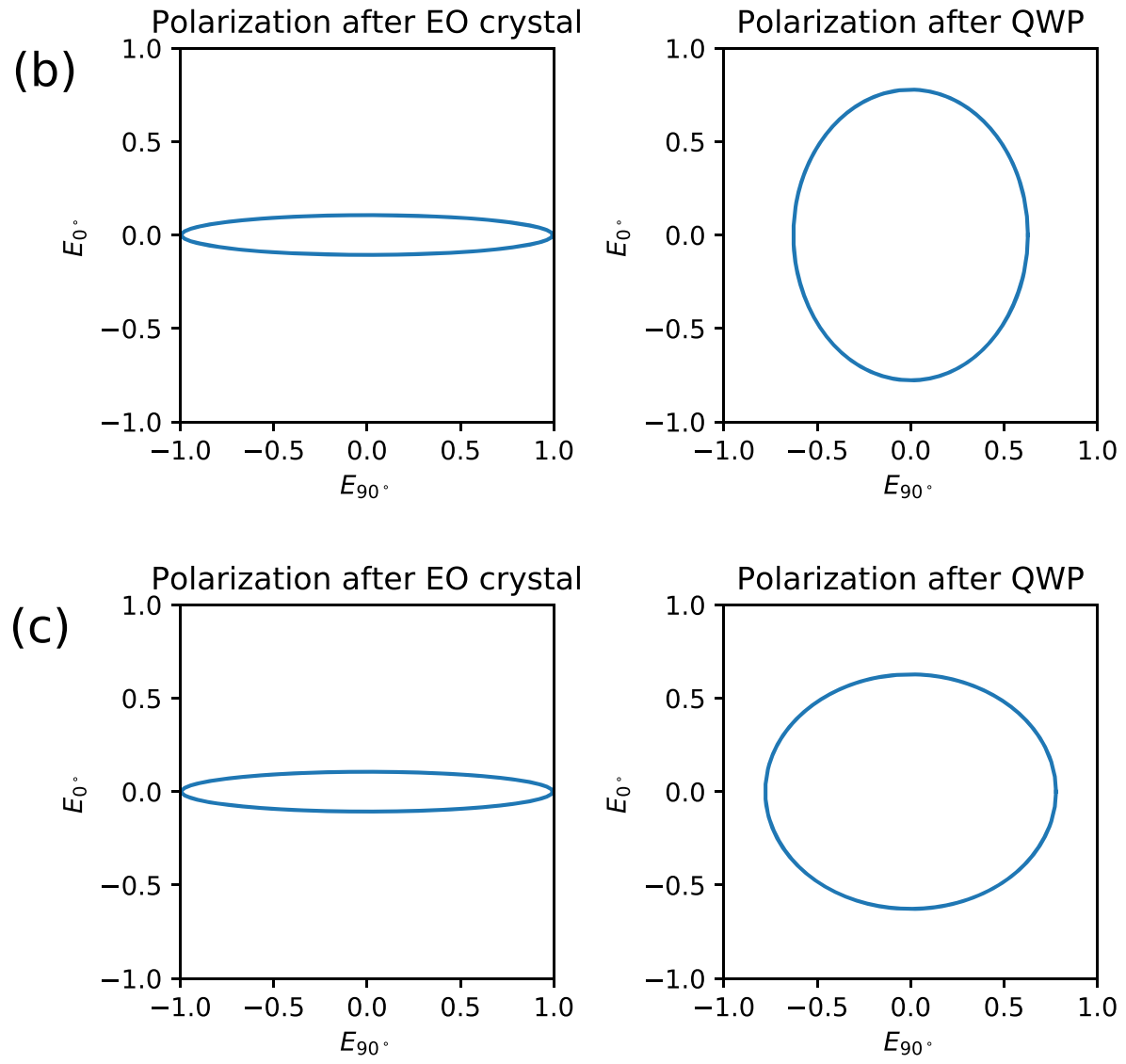

Fig. 2 a Polarizations (using electric field directions, $E_{0^{\circ}}$ and $E_{90^{\circ}}$ ) of the probe beam, without $\mathrm{THz}$. b Polarizations with $\mathrm{THz}$ causing a phase change wave plate in the EO crystal. Polarization simulations are considered with a (110) oriented $\mathrm{ZnTe}$ crystal that is $1 \mathrm{~mm}$ thick (along the direction of propagation of the $\mathrm{THz}$ and probe beams). Here $E_{T H z}=3 \mathrm{kV} / \mathrm{cm}$ is used. Plotting is explained in the discussion section. c Polarizations with $\mathrm{THz}$ causing a negative phase change wave plate in the EO crystal, with $E_{T H z}=-3 \mathrm{kV} / \mathrm{cm}$. Polarization depictions used here were first shown (partially) in [28] 

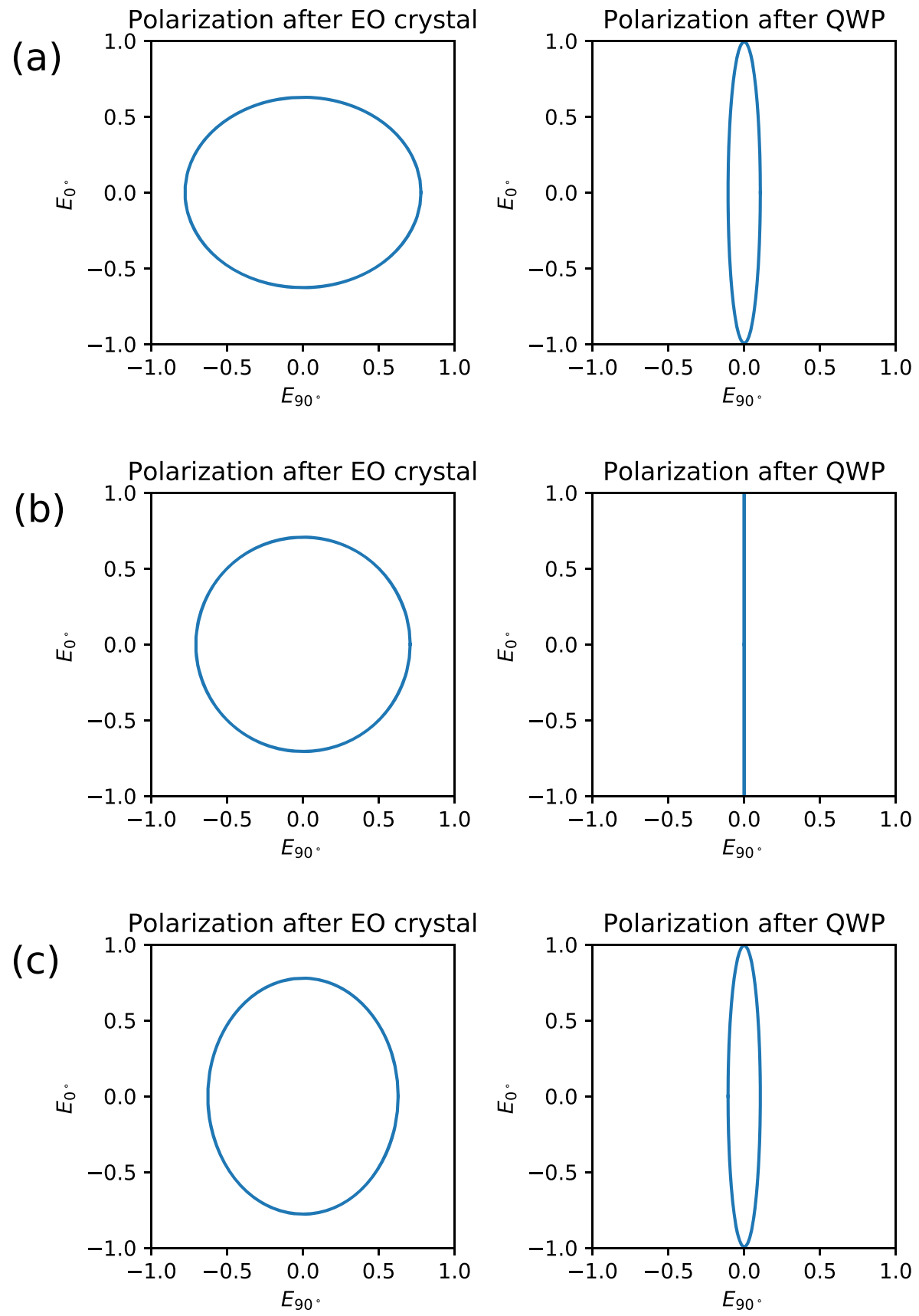

Fig. 3 a Polarizations before over-rotation with THz causing a phase change in the EO crystal, with $E_{T H z} \approx 19 \mathrm{kV} / \mathrm{cm}$. b Polarizations with THz causing a $\pi / 2$ phase change wave plate in the EO crystal, with $E_{T H z} \approx 22 \mathrm{kV} / \mathrm{cm}$. c Polarizations after over-rotation with $\mathrm{THz}$ causing phase change wave plate in the EO crystal, with $E_{T H z} \approx 25 \mathrm{kV} / \mathrm{cm}$ 

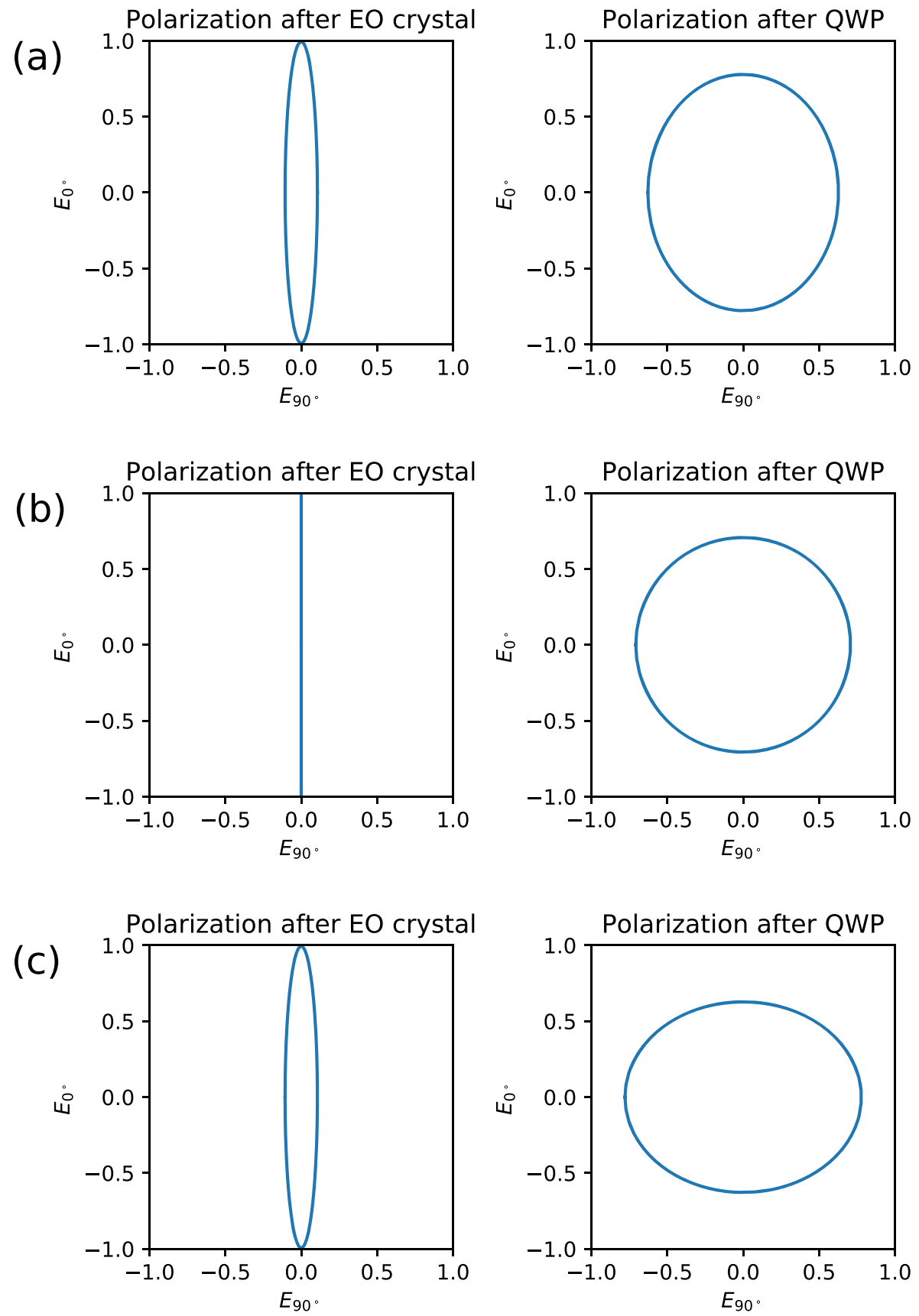

Fig. 4 a Polarizations with THz causing a phase change less than $\pi$ in the EO crystal, with $E_{T H z} \approx$ $41 \mathrm{kV} / \mathrm{cm}$. b Polarizations with THz causing a $\pi$ phase change wave plate in the EO crystal, with $E_{T H z} \approx$ $44 \mathrm{kV} / \mathrm{cm}$. c Polarizations with THz causing a phase change more than $\pi$ in the EO crystal, with $E_{T H z} \approx$ $47 \mathrm{kV} / \mathrm{cm}$ 
has reached a maximum. However, $E_{0^{\circ}}$ after the QWP is circularly polarized, and continues to decrease as $\pi$ is passed, as seen with Fig. $4 a$, c.

This method of going back and forth between information from before and/or after the QWP gives results of probe polarizations without adding any limit to the strength of the THz field measurable. The information could be garnered in a variety of ways. Scans could be taken with and without the QWP. Using data from different scans may not be ideal, but with automation a single scan could be done, with the QWP removed and replaced at each point in the movable stage used to map the THz field. Another option is the beam could be split before the QWP, going to another set of a Wollaston prism and photodiodes. One more option is a pulsed variable waveplate could be used in place of a QWP, that switches between no waveplate and QWP. In all cases, calibration would have to be done so a change in $\mathrm{THz}$ causes the same change in probe polarization intensities before and after (or with and without) the QWP.

\section{Discussion}

General intensity differences in the polarizations, to the lowest order in $\mathrm{THz}$ field, have been analysed [2]. Here we consider the special case, where the intensity difference is maximized to simulate the polarizations, $\theta_{T H z}=\theta_{\text {probe }}=90^{\circ}$. This is the typical configuration used in experiments and could easily be generalized to other configurations. To understand the polarizations, we first start with the index ellipsoid equation obtained from ZnTe in the presence of an electric field [27],

$$
\frac{x^{2}}{n_{0}^{2}}+\frac{y^{2}}{n_{0}^{2}}+\frac{z^{2}}{n_{0}^{2}}+2 r_{41} E_{T h z, x} y z+2 r_{41} E_{T H z, y} z x+2 r_{41} E_{T H z, z} x y=1
$$

where $r_{41}=3.9 \mathrm{pm} / \mathrm{V}$ [1] is the electro-optic coefficient of ZnTe, and $n=2.8528$ [29] is the index of refraction of ZnTe at $800 \mathrm{~nm}$. Using the coordinates as shown in Fig. 5, and taking $n_{0}=n, E_{T H z, z}=0$, and $-E_{T H z, x}=E_{T H z, y}=\frac{1}{\sqrt{2}} E_{T H z}$, we then obtain,

$$
\frac{x^{2}+y^{2}+z^{2}}{n^{2}}+\sqrt{2} r_{41} E_{T H z} z(y-x)=1
$$

To reduce the number of mixed terms, we can use a new system of axes, rotating around the $z$-axis $45^{\circ}$, and then a $45^{\circ}$ axis rotation around the $x^{\prime}$-axis, with,

$$
\begin{aligned}
& x=\frac{1}{\sqrt{2}}\left(x^{\prime}-\frac{1}{\sqrt{2}}\left(y^{\prime}-z^{\prime}\right)\right) \\
& y=\frac{1}{\sqrt{2}}\left(x^{\prime}+\frac{1}{\sqrt{2}}\left(y^{\prime}-z^{\prime}\right)\right) \\
& z=\frac{1}{\sqrt{2}}\left(y^{\prime}+z^{\prime}\right)
\end{aligned}
$$

which gives,

$$
\frac{x^{\prime 2}}{n^{2}}+y^{\prime 2}\left(\frac{1}{n^{2}}+r_{41} E_{T H z}\right)+z^{\prime 2}\left(\frac{1}{n^{2}}-r_{41} E_{T H z}\right)=1
$$




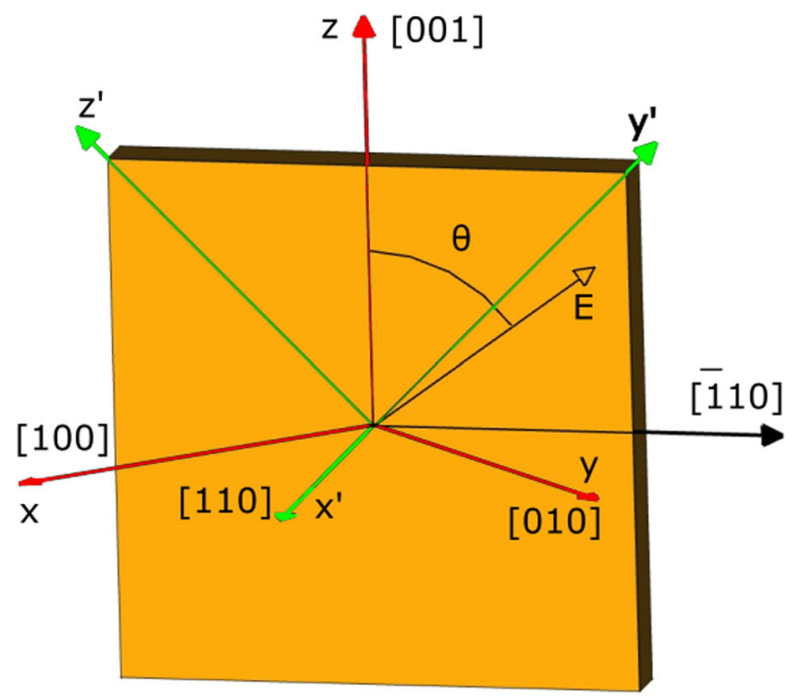

Fig. 5 (110) oriented ZnTe crystal with $x, y$, and $z$ axes, $x^{\prime}, y^{\prime}$, and $z^{\prime}$ axes, and E angle $\theta$

The result of these axis rotations is shown in Fig. 5.

When $r_{41} E_{T H z}<<\frac{1}{n^{2}}$ the approximations (right expressions),

$$
\begin{aligned}
& n_{y^{\prime}}=\frac{n}{\sqrt{1+r_{41} n^{2} E_{T H z}}} \approx n-\frac{n^{3}}{2} r_{41} E_{T H z} \\
& n_{z^{\prime}}=\frac{n}{\sqrt{1-r_{41} n^{2} E_{T H z}}} \approx n+\frac{n^{3}}{2} r_{41} E_{T H z}
\end{aligned}
$$

are commonly used [27]. However, these relations would not be valid as $E_{T H z}$ gets to be a few hundred $\mathrm{MV} / \mathrm{cm}$ - thus, we will give the more general results and include approximations when appropriate. These directionally dependent indexes of refraction can then be put into the electric field wave equations for the probe beam components, as (with the components of the probe beams at $\theta_{E_{y^{\prime}}}=45^{\circ}$ and $\left.\theta_{E_{z^{\prime}}}=-45^{\circ}\right)$,

$$
\begin{aligned}
& E_{y^{\prime}}=\sqrt{\frac{I_{p}}{2}} e^{i\left(\omega t-\left(\frac{\omega}{c}\right) n_{y^{\prime}} L\right)} \\
& E_{z^{\prime}}=\sqrt{\frac{I_{p}}{2}} e^{i\left(\omega t-\left(\frac{\omega}{c}\right) n_{z^{\prime}} L\right)}
\end{aligned}
$$

where $\omega$ is the probe beam angular frequency, $c$ is the speed of light, $t$ is time, and $L$ is the depth of the crystal in the $x^{\prime}$ direction.

However, squaring and subtracting these field directions do not give the polarization intensity differences we are looking for, $I_{\theta=0}-I_{\theta=90^{\circ}}=\Delta I$. These intensities can be obtained from Stoke's parameter $S_{2}$ (or $U$ ), as [30],

$$
\Delta I=S_{2}=U=\left|E_{y^{\prime}+45^{\circ}}\right|^{2}-\left|E_{y^{\prime}-45^{\circ}}\right|^{2}=\left\langle E_{y^{\prime}} E_{z^{\prime}}{ }^{*}\right\rangle+\left\langle E_{z^{\prime}} E_{y^{\prime}}{ }^{*}\right\rangle
$$


This then gives, along with using Eq. (5) for the approximations,

$$
\Delta I_{\text {Before } Q W P}=I_{p} \cos \left(\frac{\omega L}{c}\left(n_{y^{\prime}}-n_{z^{\prime}}\right)\right) \cong I_{p} \cos \left(\frac{\omega}{c} n^{3} r_{41} E_{T H z} L\right)
$$

With the addition of a QWP at $\theta_{Q W P}=45^{\circ}$, we would use $e^{\frac{i \pi}{4}}\left(\begin{array}{cc}1 & 0 \\ 0 & -i\end{array}\right)\left(\begin{array}{c}E_{y^{\prime}} \\ E_{z^{\prime}}\end{array}\right)$ with Eq. (8) and obtain, along with the approximation (5),

$$
\Delta I_{A f t e r} Q W P=I_{p} \sin \left(\frac{\omega L}{c}\left(n_{y^{\prime}}-n_{z^{\prime}}\right)\right) \cong I_{p} \sin \left(\frac{\omega}{c} n^{3} r_{41} E_{T H z} L\right)
$$

For a small argument of the sine, this gives the standard low $\mathrm{THz}$ field result, where the linearity of $\Delta I_{A f t e r} \propto E_{T H z}$ is commonly used (see equation 9 in [2]). The small field linearity does not hold up for high fields. In general, we would have (equation 8 in [2])

$$
\Delta I_{\text {After } Q W P}=I_{p} \sin \left(2\left(\theta_{\text {probe }}-\phi\right) \sin \left(\frac{\omega L}{c}\left(n_{y^{\prime}}\left(\theta_{T H z}\right)-n_{z^{\prime}}\left(\theta_{T H z}\right)\right)\right)\right.
$$

(of which Eq. (10) here is a special case of). In the expressions above, we assumed $\varphi_{\text {Planken et al. }}=\theta_{\text {probe }}=90^{\circ}, \alpha_{\text {Planken et al. }}=\theta_{T H z}=90^{\circ}$, and $\theta_{\text {Planken et al. }}=$ $\phi=45^{\circ}$ (with respect to the horizontal axis, $\phi=90^{\circ}-\theta$ ). This is the configuration which gives the maximum perpendicular polarization intensity change of a linearly polarized beam with a waveplate, where the waveplate fast axis would be at $45^{\circ}$ from the polarized beam angle. Thus, the first sine would be equal to 1 . The purpose of the derivation above for the special case $\theta_{T \mathrm{~Hz}}=\theta_{\text {probe }}=90^{\circ}$ is to show where Eq. (9) comes from and to illustrate the result of axes rotations.

The parametric plots of the electric field polarizations are obtained by using $e^{-i \omega t}$ and the horizontally polarized Jones vector $\left(\begin{array}{l}1 \\ 0\end{array}\right)$. The general equations for waveplates with a fast axis at an angle $\phi$, and phase retardation $\Gamma$ are then [31],

$$
e^{-\frac{i \Gamma}{2}}\left(\begin{array}{ll}
\cos ^{2} \phi+e^{i \Gamma} \sin ^{2} \phi & \left(1-e^{i \Gamma}\right) \cos \phi \sin \phi \\
\left(1-e^{i \Gamma}\right) \cos \phi \sin \phi & \sin ^{2} \phi+e^{i \Gamma} \cos ^{2} \phi
\end{array}\right)
$$

where $t$ is taken over a full wavelength. The phase for the ZnTe crystal with a fast axis at $\phi=45^{\circ}$ is

$$
\Gamma_{Z n T e}=\frac{\omega}{c} \Delta n L \cong \frac{\omega}{c} n^{3} r_{41} E_{T H z} L
$$

and a QWP at $\phi=45^{\circ}$ has a phase,

$$
\Gamma_{Q W P}=\pi / 2 \text {. }
$$

For a length $L=1 \mathrm{~mm}$, over-rotation occurs at around $22 \mathrm{kV} / \mathrm{cm}$ as can be seen in Fig. 3. Reflection of $\mathrm{THz}$ on the $\mathrm{ZnTe}$ crystal can also be taken into account [9] (frequency dependence is ignored in this reference), aside from also taking the detector response function into account [25].

With lower $\mathrm{THz}$ field, the sine in $\Delta I_{\text {After }} Q W P$ is usually taken in its first-order approximation [2]. With higher fields this would no longer be possible. Figure 6 shows increasing $E_{T H z}$ signal strengths at increasing probe and $\mathrm{THz}$ angles, using Eq. (11). The angle $\theta$ of concern here occurs at $\pi / 2$. The peak amplitude increases until over-rotation at around $22 \mathrm{kV} / \mathrm{cm}$, then decreases, becoming negative at values above $44 \mathrm{kV} / \mathrm{cm}$ - where a $\pi$ phase change occurs. This would correspond to the dips 


\section{Increasing $E_{T H z}$ field strength plots for $E_{T H z} /$ probe angles}

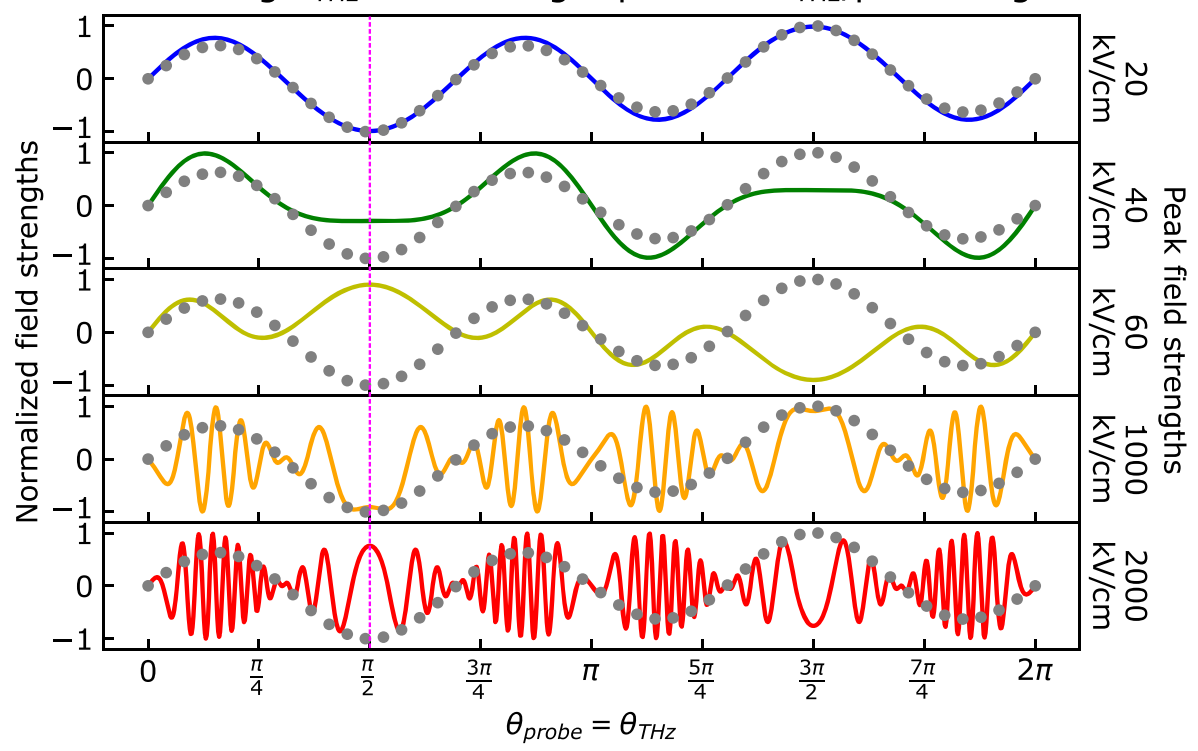

Fig. 6 Different magnitudes of $E_{T H z}$ using Eq. (11). The grey dotted lines are normalized plots using the low field approximation, where the second sine term in Eq. (11) is taken to be its argument—which would increase linearly with higher fields if it were not normalized. The vertical axis -1 to 1 values show relative peaks strengths for the given $E_{T H z}$ field strengths at $\theta_{\text {probe }}$ angles. The QWP in this setup also rotates so its fast axis always $45^{\circ}$ from $\theta_{\text {probe }}$

in the peaks going to the negative of their initial values, as explained below and shown in Fig. 7. At higher fields (1-2 MV/cm) using $E_{T H z}$ horizontal $($ at $\pi / 2)$ is more likely to give accurate results, since this angle shows a smaller rate of change in the response (see Fig. 6), though the envelope becomes more filled as the field strength continues to increase. While there is no theoretical limitation on field strength measurements introduced here, a practical concern is that at higher fields, even values around the $\pi / 2$ term change more. This could cause an error in the field strength measurement up to the amount that causes an over-rotation. However, at higher fields, this term becomes relatively smaller. Checking the precise angle would be a experimental concern. The error in the field strength measurement would also increase in absolute terms at higher fields if the angle is off of $\pi / 2$. If the angle were known precisely, corrections could be made with equations, however, if this were the case then $\pi / 2$ could just be selected. A error of the angle could give an error in the peak field measured. Also, due to $n_{x^{\prime}}=\left(\frac{1}{n^{2}}+r_{41} E_{T H z} \cos \theta_{\text {probe }}\right)^{-0.5}$ in the index ellipsoid (equation 5 in Planken et al. [2]),

$$
\begin{aligned}
x^{\prime 2}\left(\frac{1}{n^{2}}+r_{41} E_{T H z} \cos \theta_{\text {probe }}\right) & +y^{\prime 2}\left(\frac{1}{n^{2}}+r_{41} E_{T H z}\left(\cos \theta_{\text {probe }} \sin ^{2} \phi+\cos \left(\theta_{\text {probe }}+2 \phi\right)\right)\right) \\
& +z^{\prime 2}\left(\frac{1}{n^{2}}-r_{41} E_{T H z}\left(\cos \theta_{\text {probe }} \cos ^{2} \phi-\cos \left(\theta_{\text {probe }}+2 \phi\right)\right)\right)=1
\end{aligned}
$$




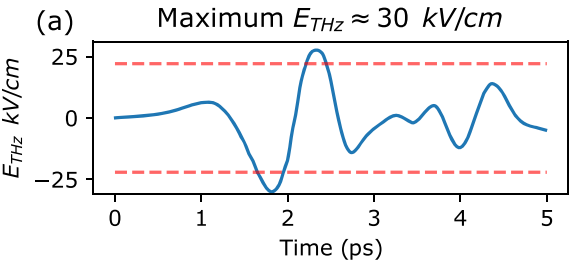

(c) Results without arcsin taken

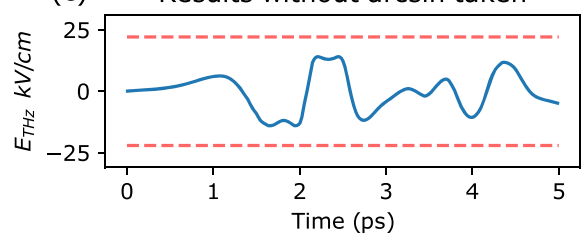

(e)

Results with arcsin taken

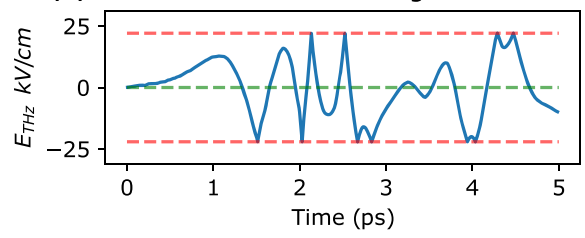

(b)

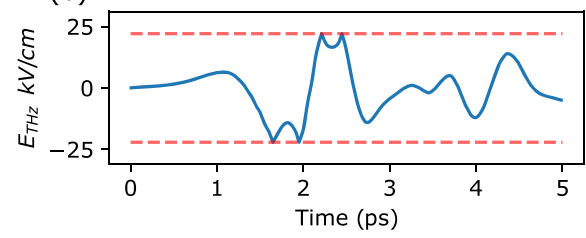

(d) Results before QWP with arccos taken

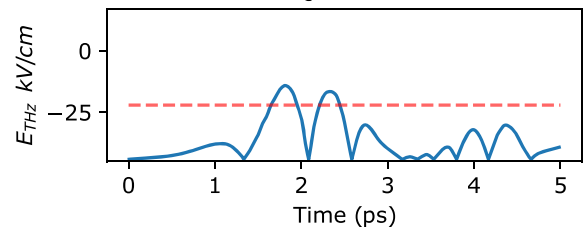

(f)

Results before QWP with arccos taken $\times 2$ field strength

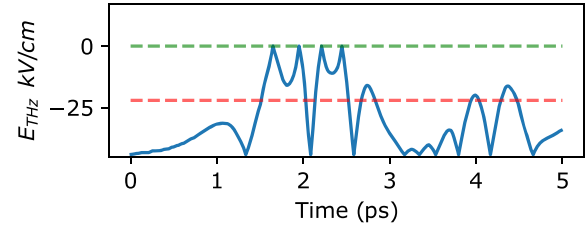

Fig. 7 a Digitized data taken from Ibrahim et al. [3], normalized to an over-rotation occurring at $E_{T H z} \approx$ $22 \mathrm{kV} / \mathrm{cm}$ (dashed red lines show this value $+/-$ in the plots). Results are displayed after the QWP showing over-rotation $\mathbf{b}$ with and $\mathbf{c}$ without the arcsin taken. $\mathbf{d}$ Results before the QWP are shown with the arccos taken. Keeping with the same positive/negative convention as in Fig. 4, a horizontal linear electric field gives the maximum magnitude negative value of $E_{T H z} \approx 44 \mathrm{kV} / \mathrm{cm}$, where values start. This could be re-calibrated to 0 in practice since it is the change that corrects for the field after the QWP. e and $\mathbf{f}$ show double the fields strength of $\mathbf{b}$ and $\mathbf{d}$, respectively. The green line shows where the second over-rotation occurs, at a $\pi$ phase change. The original waveform can be pieced together from the results of these two graphs, having evidence of where over-rotations occur

(which is a general version of Eq. (4) here, with $\phi=45^{\circ}$ giving the last index ellipse rotation), higher fields as "small" as $4 \mathrm{GV} / \mathrm{cm}$ would have an effect on phase matching as the field changes if $E_{T H z}$ is not horizontal, $\theta_{T H z}=90^{\circ}$. Thus, having the laser probe and $E_{T H z}$ horizontal to a vertical $\mathrm{ZnTe}$ [001] axis is the best option at high fields.

By not ignoring the full sine or cosine in either $\Delta I$, intensity differences could give accurate $E_{T H z}$ values at all current ranges. Also, if over-rotation has occurred and the cosine is used with $\Delta I_{\text {Before }} Q W P$, and a phase change of $\pi$ has not been reached, this could give $E_{T H z}$ in one direction for each peak. In THz science, positive and negative $\mathrm{THz}$ fields are generally mixed to take a Fourier transform of the results. Although samples being examined generally have inversion symmetry in the direction of the positive and negative $\mathrm{THz}$ fields, this still misses an opportunity to scrutinize $\mathrm{THz}$ field direction interactions separately. Thus, taking the Fourier transform of $\mathrm{THz}$ field directions separately yields more precise information on a sample, 
and avoids having to mix data sets even when over-rotation occurs. Though when $\mathrm{THz}$ fields are high enough that more than a $\pi$ phase change occurs, then both data sets before and after the QWP would have to be used.

We now apply our results to data digitized from Ibrahim et al. [3] and shown in Fig. 7. For better comparison we use their calculated over-rotation $E_{T H z} \approx 47 \mathrm{kV} / \mathrm{cm}$ normalized to the over-rotation calculated here, at $E_{T H z} \approx 22 \mathrm{kV} / \mathrm{cm}$. Thus, their maximum field at $E_{T H z} \approx 64 \mathrm{kV} / \mathrm{cm}$ occurs at $E_{T H z} \approx 30 \mathrm{kV} / \mathrm{cm}$ here. The phases are then taken with Eq. (13). Equation (12) for ZnTe and QWP waveplates is then used with horizontal polarization $\left(\begin{array}{l}1 \\ 0\end{array}\right)$ to find $\Delta I$. Finally, Eqs. (10) (with and without the arcsine argument) and (9) are solved for $E_{T H z}$, and the resulting data is plotted. The results before the QWP show definitive evidence that over-rotation occurs, since the dips in Fig. 7b do not show up in Fig. 7d. The results that leave out the arcsin show incorrect peak values (Fig. 7c), and distorted waveforms-more so in parts closer to over-rotation. The results with the arcsin taken can then be corrected to return the original $E_{T H z}$ values. This is further shown by Fig. 7e, f. The field is doubled for illustrative purposes. A second over-rotation occurs at 0 where the phase change has reached $\pi$ (see Fig. 4). Again, the original waveform can be patched together from these results, which have evidence of where over-rotations occur. This would get more complicated to see as the field gets higher and more over-rotations occur, though an automated method to correct the waveform could be made.

Over-rotation can be tested by decreasing the $\mathrm{THz}$ field with an absorber such as silicon to avoid reverse polarizations of over-rotation. This would give clear evidence that over-rotation occurs at the dip of the peaks (as seen in Fig. 7b) when the wafers are not used, where the polarization intensities reverse, even though the field is higher than the data yields [3]. The data at the dip in the peak could then be flipped - using values taken before the QWP and Eq. (9) - turning it into an uninterrupted peak instead, recreating the original field as seen in Fig. 7a during the same time range. If decreasing the intensities were considered a copy of the high field, just at a smaller scale, data could be adjusted accordingly. However, any absorption of a $\mathrm{THz}$ field has an effect on wave form, aside from just field strength, along with indexes of refraction not being uniform across the spectrum. Thus, THz field interactions with the probe in the EO crystal would not be the same at low and high fields. Even more, this would only be of any use in cases where a reference $\mathrm{THz}$ signal could be taken with absorbers that can be removed (assuming over-rotation would not occur with a sample in place, or else this method would not work in this case either), unless the absorbers occur after the sample. Of course, lowering the intensity of a $\mathrm{THz}$ field before a sample would defeat the purpose of having a high field $\mathrm{THz}$ system. Another way to avoid over-rotation would be to decrease the $\mathrm{THz}$ peak field detection by changing the $E_{T \mathrm{~Hz}}$ and/or probe angles, decreasing the signal to noise ratio and dynamic range-which is generally not preferred [32]. Trying different angle parameters with a probe polarization code can vary results under different circumstances. This would mean that Eq. (10) could not be used and reverting back to the general angle case (Eq. (11)) from Planken et al. would be necessary [2]. Thus, using the maximized detection angles - with both the probe and $\mathrm{THz}$ horizontal-is the preferable option, while avoiding over-rotation with the methods described in this article. No increase in the noise floor is presented with the methods described here; 
thus, the dynamic range would increase proportionally with higher fields. Checking that the noise floor is consistent before and after the QWP would be an experimental consideration.

As shown above, by having the complete polarization depictions of EO sampling (which, to the best of our knowledge, has not been shown correctly before), this enables us to understand the corrections to perform in case of over-rotation at high fields. In particular, we can use all polarization data from before and after the QWP, along with the proper calculations (using Eqs. (9) and (10), solved for $E_{T H z}$ ), to obtain an accurate reading of the $\mathrm{THz}$ fields even at high fields by replacing $\mathrm{THz}$ field value results after the QWP with calibrated results from before the QWP, where over-rotations occur.

\section{References}

1. Susan L Dexheimer. Terahertz spectroscopy: principles and applications. CRC press, 2007.

2. Paul CM Planken, Han-Kwang Nienhuys, Huib J Bakker, and Tom Wenckebach. Measurement and calculation of the orientation dependence of terahertz pulse detection in znte. JOSA B, 18(3):313-317, 2001.

3. Akram Ibrahim, Denis Férachou, Gargi Sharma, Kanwarpal Singh, Marie Kirouac-Turmel, and Tsuneyuki Ozaki. Ultra-high dynamic range electro-optic sampling for detecting millimeter and sub-millimeter radiation. Scientific reports, 6:23107, 2016.

4. D. You, R. R. Jones, P. H. Bucksbaum, and D. R. Dykaar. Generation of high-power sub-single-cycle 500-fs electromagnetic pulses. Opt. Lett., 18(4):290-292, Feb 1993.

5. E. Budiarto, J. Margolies, S. Jeong, J. Son, and J. Bokor. High-intensity terahertz pulses at 1-khz repetition rate. IEEE Journal of Quantum Electronics, 32(10):1839-1846, Oct 1996.

6. Ki-Yong Kim, James H Glownia, Antoinette J Taylor, and George Rodriguez. Terahertz emission from ultrafast ionizing air in symmetry-broken laser fields. Optics Express, 15(8):4577-4584, 2007.

7. Jeroen van Tilborg, CB Schroeder, Cs Tóth, CGR Geddes, Eric Esarey, and WP Leemans. Single-shot spatiotemporal measurements of high-field terahertz pulses. Optics letters, 32(3):313-315, 2007.

8. T Bartel, P Gaal, K Reimann, Michael Woerner, and Thomas Elsaesser. Generation of single-cycle thz transients with high electric-field amplitudes. Optics Letters, 30(20):2805-2807, 2005.

9. H Hirori, A Doi, F Blanchard, and K Tanaka. Single-cycle terahertz pulses with amplitudes exceeding $1 \mathrm{mv} / \mathrm{cm}$ generated by optical rectification in linbo 3. Applied Physics Letters, 98(9):091106, 2011.

10. Mengkun Liu, Harold Y Hwang, Hu Tao, Andrew C Strikwerda, Kebin Fan, George R Keiser, Aaron J Sternbach, Kevin G West, Salinporn Kittiwatanakul, Jiwei Lu, et al. Terahertz-field-induced insulatorto-metal transition in vanadium dioxide metamaterial. Nature, 487(7407):345, 2012.

11. Mostafa Shalaby, Carlo Vicario, and Christoph P Hauri. Simultaneous electronic and the magnetic excitation of a ferromagnet by intense thz pulses. New Journal of Physics, 18(1):013019, 2016.

12. Ryusuke Matsunaga, Yuki I Hamada, Kazumasa Makise, Yoshinori Uzawa, Hirotaka Terai, Zhen Wang, and Ryo Shimano. Higgs amplitude mode in the bcs superconductors nb 1- x ti x n induced by terahertz pulse excitation. Physical review letters, 111(5):057002, 2013.

13. Ryusuke Matsunaga, Naoto Tsuji, Hiroyuki Fujita, Arata Sugioka, Kazumasa Makise, Yoshinori Uzawa, Hirotaka Terai, Zhen Wang, Hideo Aoki, and Ryo Shimano. Light-induced collective pseudospin precession resonating with higgs mode in a superconductor. Science, 345(6201):1145-1149, 2014.

14. Xian Li, Tian Qiu, Jiahao Zhang, Edoardo Baldini, Jian Lu, Andrew M Rappe, and Keith A Nelson. Terahertz field-induced ferroelectricity in quantum paraelectric srtio3. Science, 364(6445):10791082, 2019.

15. TF Nova, AS Disa, Michael Fechner, and Andrea Cavalleri. Metastable ferroelectricity in optically strained srtio3. Science, 364(6445):1075-1079, 2019.

16. Hadi Razavipour, Wayne Yang, Abdeladim Guermoune, Michael Hilke, David G Cooke, Ibraheem Al-Naib, Marc M Dignam, François Blanchard, Hassan A Hafez, Xin Chai, et al. High-field response of gated graphene at terahertz frequencies. Physical Review B, 92(24):245421, 2015. 
17. Ali Mousavian, Byounghwak Lee, Andrew D. Stickel, and Yun-Shik Lee. Ultrafast photocarrier dynamics in single-layer graphene driven by strong terahertz pulses. J. Opt. Soc. Am. B, 35(6):1255-1259, Jun 2018.

18. M. B. Agranat, O. V. Chefonov, A. V. Ovchinnikov, S. I. Ashitkov, V. E. Fortov, and P. S. Kondratenko. Damage in a thin metal film by high-power terahertz radiation. Phys. Rev. Lett., 120:085704, Feb 2018.

19. Abel Woldegeorgis, Takayuki Kurihara, Mohammed Almassarani, Burgard Beleites, Ronny Grosse, Falk Ronneberger, and Amrutha Gopal. Multi-mv/cm longitudinally polarized terahertz pulses from laser-thin foil interaction. Optica, 5(11):1474-1477, Nov 2018.

20. Kensuke Teramoto, Shigeki Tokita, Tokinori Terao, Shunsuke Inoue, Ryo Yasuhara, Takeshi Nagashima, Sadaoki Kojima, Junji Kawanaka, Kazuaki Mori, Masaki Hashida, and Shuji Sakabe. Half-cycle terahertz surface waves with mv/cm field strengths generated on metal wires. Applied Physics Letters, 113(5):051101, 2018.

21. C Vicario, C Ruchert, and CP Hauri. High field broadband thz generation in organic materials. Journal of Modern Optics, 62(18):1480-1485, 2015.

22. C. Vicario, A. V. Ovchinnikov, S. I. Ashitkov, M. B. Agranat, V. E. Fortov, and C. P. Hauri. Generation of 0.9-mj thz pulses in dstms pumped by a cr:mg2sio4 laser. Opt. Lett., 39(23):6632-6635, Dec 2014.

23. C. Vicario, M. Jazbinsek, A. V. Ovchinnikov, O. V. Chefonov, S. I. Ashitkov, M. B. Agranat, and C. P. Hauri. High efficiency thz generation in dstms, dast and oh1 pumped by cr:forsterite laser. Opt. Express, 23(4):4573-4580, Feb 2015.

24. Carlo Vicario, Mostafa Shalaby, and Christoph P Hauri. Subcycle extreme nonlinearities in gap induced by an ultrastrong terahertz field. Physical review letters, 118(8):083901, 2017.

25. Yun-Shik Lee. Principles of terahertz science and technology, volume 170. Springer Science \& Business Media, 2009.

26. Kai-Erik Peiponen, Axel Zeitler, and Makoto Kuwata-Gonokami. Terahertz spectroscopy and imaging, volume 171. Springer, 2012.

27. Amnon Yariv. Quantum electronics, 3rd. Edn.(John WieLy \& Sons, New York, 1988) p, 389, 1989.

28. Gregory Bell. A terahertz emission spectrometer for the study of ultrafast carrier dynamics in semiconductors. Master's thesis, McGill University Libraries, 2017.

29. DTF Marple. Refractive index of znse, znte, and cdte. Journal of Applied Physics, 35(3):539-542, 1964.

30. Edward Collett. Field guide to polarization, volume 15. SPIE press Bellingham, 2005.

31. José J Gil and Eusebio Bernabeu. Obtainment of the polarizing and retardation parameters of a nondepolarizing optical system from the polar decomposition of its mueller matrix. Optik, 76(2):67-71, 1987.

32. Mira Naftaly and Richard Dudley. Methodologies for determining the dynamic ranges and signal-tonoise ratios of terahertz time-domain spectrometers. Optics letters, 34(8):1213-1215, 2009.

Publisher's Note Springer Nature remains neutral with regard to jurisdictional claims in published maps and institutional affiliations. 